\title{
Effects of Elastin-Like Peptide on Regulation of Human Mesenchymal Stem Cell Behavior
}

\author{
Elizabeth Jin $^{1,2}$ • Patrick T. Lee ${ }^{1}$ - Won Bae Jeon ${ }^{3}$. \\ Wan-Ju Li ${ }^{1,2}$
}

Received: 14 January 2016 /Revised: 31 March 2016 / Accepted: 6 June 2016 / Published online: 6 July 2016

(C) The Regenerative Engineering Society 2016

\begin{abstract}
Mesenchymal stem cells (MSCs) have the ability to proliferate and differentiate into different cell types. However, cell behavior has been difficult to fully control. As more is learned about how the surrounding microenvironment in the culture influences cell behavior, the importance of substrate structure and chemistry are realized. Many extracellular matrix proteins have been examined to determine their effects on regulation of cell behavior. In this study, we examined the effect of Arg-Gly-Asp-enriched elastin-like peptide (RGD-ELP), a recombinant peptide containing both elastin repeating units and the RGD adhesion domains, on MSC adhesion, proliferation, cell morphology, and differentiation. To this end, cells were seeded on monolayer or nanofibrous substrates that were coated without any molecule as a negative control, with RGD-ELP, or with fibronectin as a positive control. DNA content was measured 6 h, 3 days, and 7 days after cell seeding. Our results showed that RGD-ELP-coated monolayer substrates were
\end{abstract}

Won Bae Jeon

wbjeon@dgist.ac.kr

$\triangle \quad$ Wan-Ju Li

li@ortho.wisc.edu

1 Musculoskeletal Biology and Regenerative Medicine Laboratory, Department of Orthopedics and Rehabilitation, School of Medicine and Public Health, University of Wisconsin-Madison, 1111 Highland Avenue, WIMR 5051, Madison, WI 53705-2275, USA

2 Department of Biomedical Engineering, College of Engineering, University of Wisconsin-Madison, Madison, WI, USA

3 Laboratory of Biochemistry and Cellular Engineering, Division of NanoBio Technology, Daegu Gyeongbuk Institute of Science and Technology, Daegu 711-873, South Korea able to support cell proliferation, whereas RGD-ELPcoated nanofibrous substrates were able to enhance cell adhesion but not proliferation, suggesting that substrate topography plays a role in the regulation of cell adhesion and proliferation. Cells cultured on RGD-ELP-coated monolayer or nanofibrous substrates for 2 days demonstrated a more elongated, spread morphology than cells cultured on the uncoated substrates. RGD-ELP-coated monolayer or nanofibrous substrates increased mineral formation in MSC culture compared to both control substrates. The mRNA expression of lineage-specific markers showed that MSCs cultured on RGD-ELP-coated nanofibrous substrates were increasingly induced to differentiate into the osteogenic and adipogenic lineages compared to those cultured on uncoated substrates. Overall, our results suggest that RGD-ELP coating is able to support increased cell adhesion and osteogenesis.

\section{Lay Summary}

Mesenchymal stem cells (MSCs) have been used as a clinical practice to treat patients with graft-versus-host disease. With the potential in regenerative medicine, therapeutic use of the cell recently emerges as a promising strategy to treat degenerative diseases. In fact, a number of clinical trials have been carried out to explore the potential of MSCs for disease treatment. Results of this study suggest that MSC properties can be enhanced through culturing the cell on nanofibrous substrates coated with chemically defined peptides. Our approach using a FDA-approved polymeric substrate along with chemically defined peptides to prepare MSCs for cell therapies can be considered highly translational for clinical applications.

Keywords Elastin-like peptide · Mesenchymal stem cell · Substrate topography $\cdot$ Substrate coating $\cdot$ Osteogenesis 


\section{Introduction}

Human mesenchymal stem cells (MSCs) are a promising cell source for stem cell therapy applications due to their capacity of self-renewal and trilineage differentiation [1-3]. However, in order to use MSCs for therapies, further understanding and control of MSC behavior is necessary. MSCs are capable of self-renewal for a set amount of time but will eventually undergo senescence, making it difficult to continue obtaining a large quantity of MSCs from culture for clinical use [4-6]. Additionally, senescence decreases the differentiation capability of MSCs [7, 8]. Differentiation of MSCs into a specific lineage can easily be influenced by a number of factors such as MSC source, medium component, or culture condition [9-11]. Therefore, studies investigating the influence of the extracellular environment on stem cell behavior are vitally important.

Topography of the extracellular matrix (ECM) has been shown to impact stem cell self-renewal and differentiation [12-14]. Synthetic nanofibers imitating natural ECM have been shown to differentially influence cell adhesion, proliferation, and differentiation compared to other substrates with different topographies $[15,16]$. Nanofibrous substrates have especially demonstrated the ability to enhance MSC osteogenesis [15]. Besides their impact on cell behavior, nanofibrous substrates are a viable candidate for tissue engineering substrates due to their high surface to volume ratio which facilitates proper nutrient and oxygen transfer [17, 18]. The high surface to volume ratio of the nanofiber structure supports cell growth, while the nanotopography can regulate cell behavior, making the nanofiber a promising ECM choice.

Poly(l-lactic acid) (PLLA) is a biocompatible, biodegradable synthetic polymer commonly used to fabricate nanofibrous substrates for in vivo use in orthopedic applications [19-22]. However, synthetic polymers such as PLLA lack biologically relevant adhesion sites, resulting in poor cell attachment and cell-matrix interactions [23]. To improve cellsubstrate interactions for cells cultured on synthetic polymer matrices, ECM proteins such as fibronectin (FN) can be coated on the nanofiber surface $[24,25]$. By using coated ECM proteins as a mechanism to enhance cell interactions with PLLA, it is possible to have a biologically relevant surface for cell interactions on a synthetically derived polymer substrate.

Elastin-like peptide (ELP) is a recombinant peptide which mimics the elastin ECM protein found in vivo. Previous interest in ELP has revolved around the temperature responsive nature of this peptide and its use for drug delivery applications $[26,27]$. However, since elastin is an ECM protein, recombinant ELP has recently been studied for its potential as a biomaterial substrate for cell culture support. It has been shown that ELP enhances muscle cell proliferation and differentiation [28] and MSC osteogenesis [29]. To improve biological functions of ELP, recombinant ELP incorporating the Arg-Gly-Asp (RGD) peptide has been developed [30]. The RGD peptide found in some ECM proteins is known to enhance cytocompatibility and cell attachment on synthetic substrates [31-35]. The addition of RGD to ELP has been shown to regulate cell morphology, proliferation, and differentiation [36]. While having demonstrated the effect of ELP or RGD-ELP on regulation of cell activities, these studies were performed with the peptide coated on monolayer substrates without considering the fact that cells are surrounded by the three-dimensional, nanostructural ECM in their native microenvironment. In this study, we were interested in investigating the effect of RGD-ELP together with a nanotopographic substrate on regulation of cell behavior.

Previous research has demonstrated the importance of ECM surface chemistry on directing stem cell adhesion, proliferation, and differentiation. Here, we report the impact of a recombinant ECM mimetic peptide, RGD-enriched ELP, on bone marrowderived MSC behavior. We hypothesized that the addition of our peptide to nanofibrous substrates enhances cell interactions with the synthetic matrix, which plays a role in directing stem cell proliferation and differentiation behavior.

\section{Materials and Methods}

\section{Culture of MSCs}

Ethical approval of human tissue procurement for this study was granted by the Institutional Review Board (IRB) at the University of Wisconsin-Madison. Bone marrow-derived MSCs were harvested from femoral heads and necks of patients undergoing total hip arthroplasty and were cultured in Dulbecco's modified Eagle medium (DMEM; Gibco, Grand Island, NY) supplemented with $10 \%$ fetal bovine serum (FBS; Invitrogen, Carlsbad, CA) and $1 \%$ antibiotics (10, $000 \mathrm{IU} / \mathrm{mL}$ penicillin, $10,000 \mu \mathrm{g} / \mathrm{mL}$ streptomycin, and $25 \mu \mathrm{g} / \mathrm{mL}$ amphotericin B; Mediatech, Manassas, VA). Medium changes occurred every 3 days, and the culture was maintained at $37{ }^{\circ} \mathrm{C}$ in a $95 \%$ humidified environment with $5 \% \mathrm{CO}_{2}$. Cells were expanded until they reached $80 \%$ confluence and then passaged using $0.05 \%$ trypsin/EDTA (Mediatech). Passaged cells were quantified with a hemocytometer and then seeded on substrates.

\section{Fabrication and Preparation of Spin-Coated and Nanofibrous Substrates}

PLLA (Polysciences Inc., Warrington, PA) was dissolved in chloroform (Fisher Scientific, Waltham, MA) and N,Ndimethylformamide (DMF; Acros Organics, Waltham, MA) at a 10:1 ratio, respectively, to prepare $12 \%$ PLLA solution by vortexing for $24 \mathrm{~h}$ at room temperature. To fabricate nanofibrous substrates, we followed the electrospinning process described in our previous report [37]. Briefly, the polymer 
solution was transferred to a $3-\mathrm{mL}$ syringe with an 18 -g needle held 18 in above the collection platform. A voltage of $20 \mathrm{kV}$ was applied to the system to induce the formation of nanofibers. Once a thin layer of nanofibers was spun onto the collection plate, 20-mm glass coverslips (Bioscience Tool, San Diego, CA) were placed on top of the mat and electrospinning was continued for an equivalent amount of time to equally coat the top side of coverslips.

Alternatively, $12 \%$ PLLA monolayer substrates were fabricated with a spin-coating procedure using a spin processor (model WS650SZ6NPP/LITE, Laurell Technologies) with nitrogen (60 PSI) to pressurize the motor seal and purge the process chamber, vacuum, and condensed exhaust. The amount of $500 \mu \mathrm{L}$ of $12 \%$ PLLA solution was applied to a stationary 20-mm-diameter glass coverslip (Bioscience Tools) and then immediately spun at $500 \mathrm{rpm}$ for $5 \mathrm{~s}$, followed with a spin of $3000 \mathrm{rpm}$ for $30 \mathrm{~s}$. Substrates were removed from the spin processor for $5 \mathrm{~min}$ to ensure that all solvent had evaporated. Then, the procedure was repeated by coating PLLA on the other uncoated side of the substrate. Coating both sides of the glass coverslip prevented PLLA detachment from the coverslip in the presence of medium. Both electrospun and spin-coated coverslips were constructed with humidity levels maintained between 15 and $30 \%$, then stored in a desiccator.

\section{Substrate Preparation}

Both sides of the substrates were sterilized under UV light for $30 \mathrm{~min}$. Next, substrates were further sterilized in $70 \%$ ethanol (Sigma-Aldrich) for $30 \mathrm{~min}$, washed twice for $20 \mathrm{~min}$ in HyPure cell culture grade water (ThermoScientific, South Logan, UT), and soaked in phosphate-buffered saline (PBS) (ThermoScientific). Substrates were incubated at $4{ }^{\circ} \mathrm{C}$ overnight with RGD-ELP obtained from Dr. Jeon at Daegu Gyeongbuk Institute of Science and Technology through collaboration or human FN (catalog 33,016,015; Life Technologies, Grand Island, NY) at a concentration of $5 \mu \mathrm{g} /$ $\mathrm{mL}$. The amino acid sequence of RGD-ELP monomer is TGPG[VGRGD(VGVPG) $\left.{ }_{6}\right]_{20}$ WPC with the MW of 60 , $428 \mathrm{Da}$, consisting of alternating elastic (VGVPG) 6 structural domains and cell-binding VGRGD motifs [38]. Control substrates were incubated in PBS with no peptide. Solutions were incubated at $4{ }^{\circ} \mathrm{C}$ overnight to allow for protein adsorption.

\section{Seeding of MSCs onto Substrates}

Upon reaching $80 \%$ confluence, cells were trypsinized, counted, and seeded onto the substrates. MSCs were seeded at a density of $5 \times 10^{3}$ cells $/ \mathrm{cm}^{2}$ for proliferation studies and actin filament staining. A cell density of $2 \times 10^{4}$ cells $/ \mathrm{cm}^{2}$ was used for other assays. Substrates were placed into 6-well plates that had been treated with $0.5 \%$ poly(2-hydroxyethyl methacrylate) (Polysciences) to prevent cell adhesion to tissue culture plastic. Cells were then seeded onto each substrate. Plates were placed in the incubator for $6 \mathrm{~h}$ to allow cell attachment to substrates. After 6 h, $3 \mathrm{~mL}$ of culture medium was very slowly added to each well with care taken so as not to dislodge newly adhered cells. Culture medium changes occurred every 3 days.

\section{Differentiation Induction of MSCs}

DMEM culture medium containing $10 \%$ FBS was supplemented with $10^{-3} \mathrm{M}$ dexamethasone (Sigma-Aldrich), $50 \mu \mathrm{g}$ / $\mathrm{mL}$ L-ascorbic acid 2-phosphate (Sigma-Aldrich), $10 \mathrm{mM} \beta$ glycerophosphate (Sigma-Aldrich), and $0.1 \mu \mathrm{M}$ vitamin D (EnzoLife Sciences, Farmingdale, NY) to induce osteogenesis. To induce adipogenesis, culture medium was supplemented with $10^{-6} \mathrm{M}$ dexamethasone (Sigma-Aldrich), $0.5 \mathrm{mM} 3-$ isobutyl-1-methylxanthine (IBMX; Sigma-Aldrich), and $1 \mu \mathrm{g} / \mathrm{mL}$ insulin (Sigma-Aldrich). For chondrogenic induction, MSCs were cultured in high-glucose DMEM (Life Technologies), $1 \%$ antibiotics, $1 \%$ ITS + Premix $(6.25 \mu \mathrm{g} /$ $\mathrm{mL}$ insulin, $6.25 \mu \mathrm{g} / \mathrm{mL}$ transferring, $6.25 \mu \mathrm{g} / \mathrm{mL}$ selenious acid, $1.25 \mathrm{mg} / \mathrm{mL}$ bovine serum albumin, and $5.35 \mu \mathrm{g} / \mathrm{mL}$ linoleic acid; BD Biosciences), $1 \mathrm{mM}$ sodium pyruvate (Sigma), $50 \mu \mathrm{g} / \mathrm{mL}$ L-ascorbic acid 2-phosphate, $40 \mu \mathrm{g} / \mathrm{mL}$ L-proline (Fluka, St. Louis, MO), $0.1 \mu \mathrm{M}$ dexamethasone, 10 ng/mL TGF $\beta 1$ (Peprotech, Rock Hill, NJ), and 150 ng/ mL BMP7 (Peprotech). Supplemented medium was added to the culture $6 \mathrm{~h}$ after seeding. Medium changes occurred every 3 days, and success of differentiation was assessed 7, 14 , and 21 days after induction.

\section{Proliferation Assay}

To analyze MSC attachment and proliferation, an assay was performed using the Cell Titer 96 Aqueous One Solution Cell Proliferation Assay kit (Promega, Madison, WI). Cells were seeded on nanofibrous substrates at $5 \times 10^{4}$ cells $/ \mathrm{cm}^{2}$ and analyzed on days 0,5 , and 10 . To form the assay mixture, Cell Titer solution and phenol red free DMEM low-glucose medium (Invitrogen) were combined according to the manufacturer's protocol. Substrates were washed twice with HBSS (ThermoScientific). Then, the assay mixture was added to each well and incubated at $37^{\circ} \mathrm{C}$ for $1 \mathrm{~h}$. After $1 \mathrm{~h}$, samples were analyzed at $490 \mathrm{~nm}$.

\section{Cytoskeleton Staining}

MSCs were seeded on substrates and cultured for 2 days to allow for cell adhesion and spreading. After 2 days, cells were stained according to the manufacturer's protocol with phalloidin and DAPI (Life Technologies) to visualize actin and the nucleus, respectively. Briefly, substrates were washed two times with PBS, fixed for 5 min with $3.7 \%$ formaldehyde 
(16\%, methanol-free, Ultra Pure EM Grade, Polysciences Inc.), and washed extensively with PBS. Cell membranes were then made permeable with $0.1 \%$ Triton X-100 in PBS for 5 min. Substrates were once again washed extensively with PBS and then stained with $50 \mu \mathrm{g} / \mathrm{mL}$ Alexa Fluor 488 phalloidin (Life Technologies, Grand Island, NY) in PBS for 40 min at room temperature in the dark. After $40 \mathrm{~min}$, substrates were washed four times with PBS to remove any unbound stain.

Coverslips were mounted using ProLong Gold antifade reagent with DAPI (Life Technologies). Substrates were then imaged using a Nikon Eclipse E600 microscope with a Nikon Y-FL EPI Fluorescence unit. Images were analyzed using the ImageJ software. Cell aspect ratio was determined using a previously defined method in which the longest length across the cell is divided by the perpendicular width [39]. The number of 10 cells per randomly selected area of a substrate was analyzed. With three selected areas per substrate and three substrates per experimental condition, a total of 90 cells were analyzed to determine the aspect ratio. The smallest possible aspect ratio was the value of 1 , at which the cell would be equally spread in both directions.

\section{Calcium Deposition Analysis}

Alizarin Red stain (Rowley Biochemical Institute, Danvers, MA) was used to examine calcium deposition on the substrates 14 days after induction. Substrates were rinsed twice with PBS, fixed with $60 \%$ isopropanol (Sigma-Aldrich) for $1 \mathrm{~min}$, washed three times with $\mathrm{dH}_{2} \mathrm{O}$, and stained with $2 \%$ Alizarin Red stain for $5 \mathrm{~min}$. Substrates were then washed extensively with $\mathrm{dH}_{2} \mathrm{O}$ and imaged.

\section{Alkaline Phosphatase Staining}

Alkaline phosphatase (ALP) activity was determined using the Alkaline Phosphatase kit (Sigma-Aldrich) following the manufacturer's protocol. On day 14, samples were washed two times with Tris-buffered saline (TBS; Cellgro, Manassas, VA) and fixed with $2 \%$ formaldehyde in methanol for $1 \mathrm{~min}$ at $4{ }^{\circ} \mathrm{C}$. Samples were then washed three times with water and air dried for $5 \mathrm{~min}$. A staining solution composed of $2 \mathrm{mM}$ sodium nitrite, $0.1 \mathrm{mg} / \mathrm{mL}$ of FBB-alkaline solution, and $0.9 \mathrm{mg} / \mathrm{mL}$ of naphthol AS-BI alkaline solution was added to the samples and incubated in the dark for $30 \mathrm{~min}$ at $37^{\circ} \mathrm{C}$. After $30 \mathrm{~min}$, samples were imaged.

\section{Lipid Droplet Staining and Quantification}

Lipid droplets were stained using Oil Red O staining following the company protocol on day 14. Briefly, substrates were rinsed twice with PBS and fixed with $10 \%$ formalin (Fisher Scientific) for $60 \mathrm{~min}$ at room temperature. Oil Red O stain was prepared by mixing the stock solution composed of $300 \mathrm{mg}$ Oil Red O powder (Sigma-Aldrich) dissolved in $99 \%$ isopropanol (Sigma-Aldrich) with $\mathrm{dH}_{2} \mathrm{O}$ at a 3:2 ratio and filtering after $10 \mathrm{~min}$. After fixation, substrates were rinsed three times with $\mathrm{dH}_{2} \mathrm{O}$ and then incubated with $60 \%$ isopropanol for $5 \mathrm{~min}$. Oil Red $\mathrm{O}$ staining solution was added to the substrates and agitated for $5 \mathrm{~min}$. Substrates were then washed three times with $\mathrm{dH}_{2} \mathrm{O}$ to remove excess dye and imaged. Stain was extracted with $100 \%$ isopropanol and samples were analyzed at $540 \mathrm{~nm}$.

\section{Quantitative RT-PCR}

To analyze the messenger RNA (mRNA) expression of lineage specific markers, substrates were first dissolved with TRIzol (Invitrogen) according to the manufacturer's protocol. Briefly, substrates were first dissolved in TRIzol, followed by the addition of chloroform. RNA was precipitated with the addition of isopropanol and 10-min incubation. RNA was washed three times with $80 \%$ ethanol and then resuspended in RNase-free water. Complementary DNA (cDNA) was synthesized using the High-Capacity cDNA Reverse Transcriptase kit (Life Technologies). Real-time PCR analysis was conducted using an iQ SYBR Green Supermix (BioRad, Hercules, CA). Quantitative RT-PCR was set for 40 cycles to amplify DNA products. Primer sequences are listed in Table 1. Osteocalcin (OC), runt-related transcription factor 2 (RUNX2), and ALP expressions were quantified to examine the success of osteogenesis. To examine chondrogenesis, collagen type 2 (COL2), sex-determining region Y-box9 (SOX9), and aggrecan ( $A G N)$ expressions were quantified. Peroxisome proliferator-activated receptor-gamma (PPARG) and lipoprotein lipase (LPL) expressions helped determine adipogenic differentiation within cells. The housekeeping gene ubiquitin $C(U B C)$ was used as an internal control for the quantitative RT-PCR analysis.

\section{Statistical Analysis}

All quantitative studies were performed in triplicate $(n=3)$ and reported as the mean $\pm \mathrm{SD}$. Statistical significance was analyzed using one-way ANOVA. If the $p$ value was less than 0.05 , a two-tailed $t$ test was performed to further determine statistical significance. $P$ values $<0.05$ for the $t$ test were considered statistically significant and indicated in figures with an asterisk.

\section{Results}

\section{RGD-ELP Coating Enhances Cell Adhesion to Nanofibrous Substrates}

To investigate cell adhesion and proliferation on uncoated, RGD-ELP-coated, and FN-coated monolayer and nanofibrous substrates, cell numbers were measured by the MTS assay. Uncoated, RGD-ELP-coated, and FN-coated monolayer 
Table 1 Primer sequences for qRT-PCR analysis

\begin{tabular}{|c|c|c|}
\hline Gene name & Accession number & Primer sequences $\left(5^{\prime}\right.$ to $\left.3^{\prime}\right)$ \\
\hline$O C$ & NM_199173.3 & $\begin{array}{l}\text { F GACTGTGACGAGTTGGCTGA } \\
\text { R GGAAGAGGAAAGAAGGGTGC }\end{array}$ \\
\hline$R U N X 2 / C B F A 1$ & NM_004843.3 & $\begin{array}{l}\text { F GGTTCCAGCAGGTAGCTGAG } \\
\text { R AGACACCAAACTCCACAGCC }\end{array}$ \\
\hline$A L P$ & NM_000478.3 & $\begin{array}{l}\text { F CAAAGGCTTCTTCTTGCTGG } \\
\text { R GGTCAGAGTGTCTTCCGAGG }\end{array}$ \\
\hline COL2 & NM_001844.4 & $\begin{array}{l}\text { F CCTCTGCGACGACATAATCT } \\
\text { R CTCCTTTCTGTCCCTTTGGT }\end{array}$ \\
\hline$A G N$ & NM_013227.2 & $\begin{array}{l}\text { F CACGATGCCTTTCACCACGAC } \\
\text { R TGCGGGTCAACAGTGCCTATC }\end{array}$ \\
\hline SOXO & NM_000346.3 & $\begin{array}{l}\text { F TAAAGGCAACTCGTACCCAA } \\
\text { R ATTCTCCATCATCCTCCACG }\end{array}$ \\
\hline PPARG & NM_138711.3 & $\begin{array}{l}\text { F ATGACAGCGACTTGGCAATA } \\
\text { R GGCTTGTAGCAGGTTGTCTT }\end{array}$ \\
\hline$L P L$ & NM_000237.2 & $\begin{array}{l}\text { F AGGAGCATTACCCAGTGTCC } \\
\text { R GGCTGTATCCCAAGAGATGGA }\end{array}$ \\
\hline$U B C$ & NM_021009.4 & $\begin{array}{l}\text { F TGAAGACACTCACTGGCAAGACCA } \\
\text { R CAGCTGCTTTCCGGCAAAGATCAA }\end{array}$ \\
\hline
\end{tabular}

Forward and reverse primers are indicated as " $F$ " and "R," respectively substrates all had similar cell adhesion and proliferation rates (Fig. 1a). However, our data demonstrated that on nanofibrous substrates, RGD-ELP-coating enhances cell adhesion compared to cells cultured on FN-coated and uncoated nanofibrous substrates (Fig. 1b). At day 0 , significantly more cells adhered to RGD-ELP-coated nanofibrous substrates

a

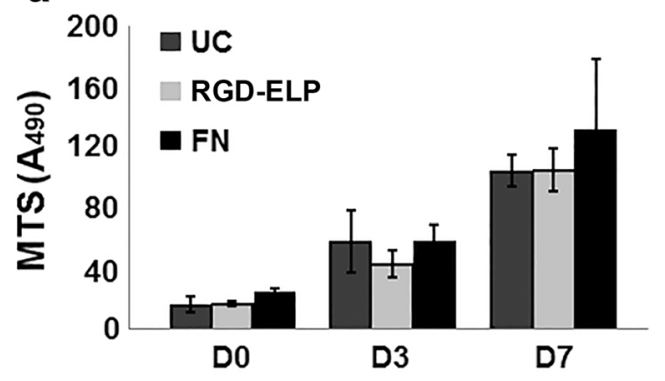

b

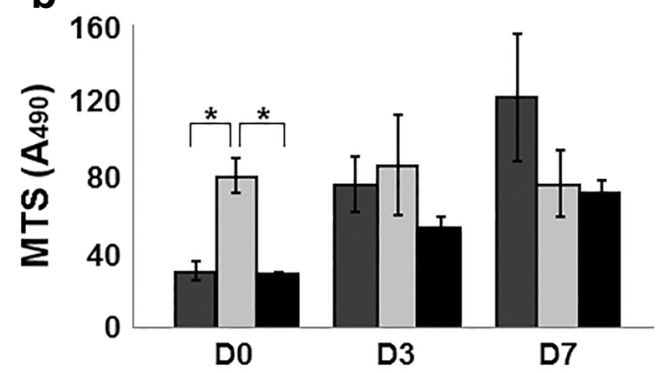

Fig. 1 Cell adhesion and proliferation quantified with the MTS assay. Cell numbers on monolayer substrates (a) or nanofibrous substrates (b) were determined after 1,3 , and 7 days of culture. ${ }^{*} p<0.05 ; n=3$. UC uncoated substrates, RGD-ELP RGD-ELP-coated substrates, $F N$ fibronectin-coated substrates compared to FN-coated substrates or uncoated substrates. Cell adhesion was increased on RGD-ELP-coated nanofibrous substrates whereas cell proliferation was not improved by the coating. By day 7, cell numbers on FN-coated nanofibrous substrates were similar to those on RGD-ELPcoated substrates. Taken together, cell adhesion was enhanced in the presence of an RGD-ELP coating combined with threedimensional nanofibrous topography.

\section{RGD-ELP Coating Supports Cell Attachment and Spreading as FN Coating}

To examine cell morphology, the actin cytoskeleton was visualized using phalloidin staining and the cell aspect ratio was determined using the ImageJ software. The acquired images demonstrated that cells cultured on uncoated substrates were significantly less elongated and had lower aspect ratios than cells cultured on RGD-ELP-coated substrates regardless of surface topography (Fig. 2). A comparison between RGDELP coating and FN coating reveals that, on monolayer substrates, cells cultured on RGD-ELP-coated substrates are significantly more elongated while cells cultured on RGD-ELPor FN-coated nanofibrous substrates have a similar morphology. Cells cultured on both RGD-ELP- and FN-coated nanofibrous substrates demonstrated a spindle-like morphology and appeared oriented along the fibers of the matrix. Based on cytoskeletal arrangement, it is clear that, in a threedimensional environment, RGD-ELP coating supports the cell morphology similar to that of cells cultured on FN. 
Fig. 2 Actin filaments visualized with phalloidin staining. MSCs were cultured on uncoated monolayer substrates (a), uncoated nanofibrous substrates (b), RGD-ELP-coated monolayer substrates (c), RGD-ELP-coated nanofibrous substrates (d), FN-coated monolayer substrates (e), and FN-coated nanofibrous substrates (f). Aspect ratios of cells cultured on monolayer substrates (g) and nanofibrous substrates (h) were calculated. Scale bar $=100 \mu \mathrm{m} . * p<0.05$; $n=3$. $U C$ uncoated substrates, $R G D$-ELP RGD-ELP-coated substrates, $F N$ fibronectin-coated substrates
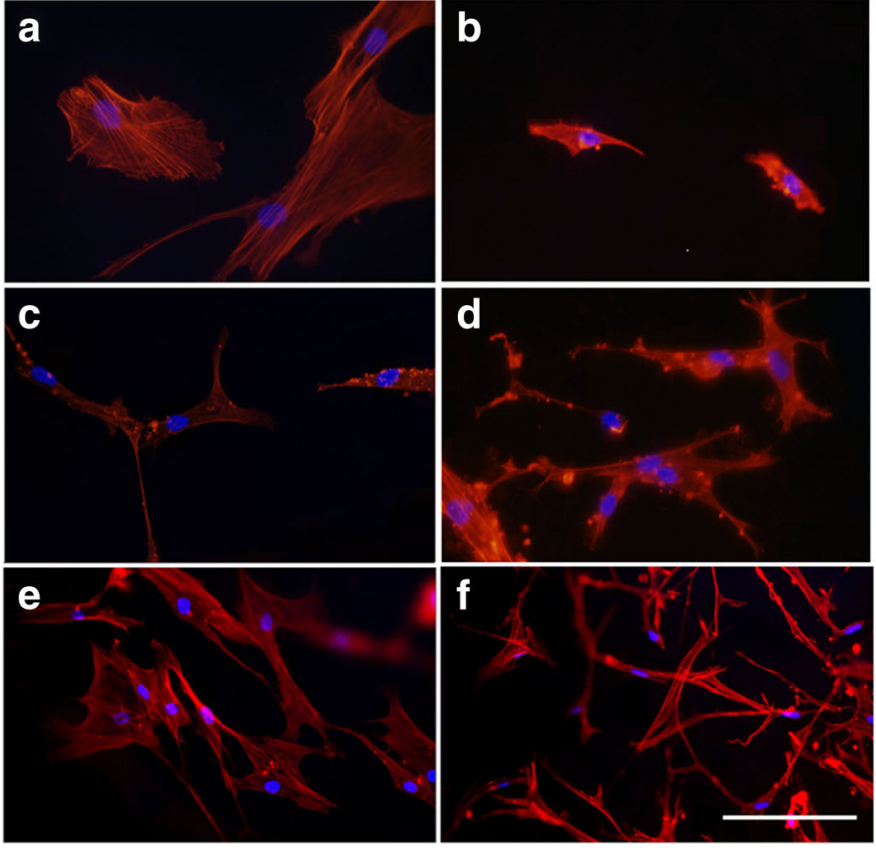

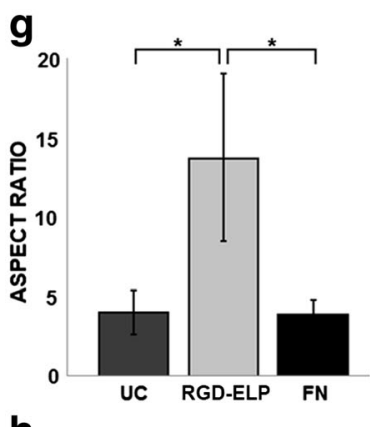

h

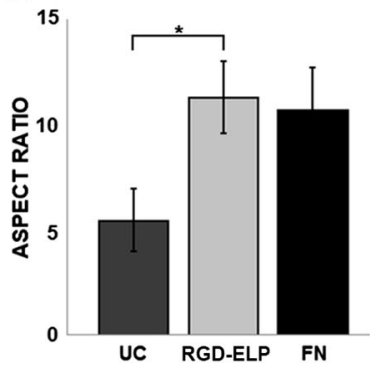

\section{RGD-ELP Coating Enhances Matrix Mineralization}

To determine effects of different coatings and surface topography, substrates were stained for ALP activity and calcium nodule formation. ALP activity was similar on all substrates regardless of the surface coating or topography (Fig. 3), indicating that the RGD-ELP coating neither enhanced nor repressed ALP activity. To further investigate the impact of surface chemistry and topography on osteogenesis, substrates were stained with Alizarin Red to examine calcium nodule formation. The Alizarin Red stain indicated the presence of calcium nodules on all substrates examined, but calcium deposition was most abundant on RGD-ELP-coated substrates compared to uncoated or FN-coated substrates (Fig. 4), suggesting that RGD-ELP plays a important role in supporting matrix mineralization. Interestingly, enhanced calcium nodule formation on RGD-ELP-coated monolayer substrates was far greater than that on RGD-ELP-coated nanofibrous substrates. This may be a result of a greater number of cells on the monolayer substrate that produced more total calcium nodules than that on the nanofibrous substrate.

\section{Adipogenic Differentiation Is Unaffected by the Presence of RGD-ELP}

MSCs were seeded on RGD-ELP-coated, FN-coated, and uncoated PLLA substrates and maintained in adipogenic medium. Lipid droplets were visualized by Oil Red O staining, and quantitative analysis of the stain was performed. Lipid droplets were found on all monolayer substrates, but Oil Red O staining did not conclusively indicate the presence of lipid droplets on the nanofibrous substrates (Fig. 5). The Oil Red O stain was then extracted and quantitatively analyzed. To control for nonspecific staining, acellular substrates were stained and quantified in the same way as seeded substrates and the OD reading subtracted out as background. Optical density analysis of the extracted stain subsequently revealed that uncoated and RGDELP-coated nanofibrous substrates had higher Oil Red O staining than FN-coated substrates (Fig. 5h). Taken together, these data demonstrate that lipid droplet accumulation is not affected by RGD-ELP coating.

\section{RGD-ELP Enhances Osteogenic Differentiation on PLLA Nanofibrous Substrates but Not Chondrogenic Differentiation}

RT-PCR of osteogenic, adipogenic, and chondrogenic mRNA transcripts was conducted for cells cultured on nanofibrous substrates to further investigate the effect of RGD-ELP coating on MSC differentiation. To determine if RGD-ELP coating enhanced osteogenesis, expressions of $O C, R U N X 2$, and $A L P$ were examined (Fig. 6a-c). $O C$ expression was significantly upregulated on RGD-ELP-coated nanofibrous substrates on days 7 and 14 compared to the uncoated nanofibrous substrate. Analysis of RUNX2 showed that early expression was highest on uncoated nanofibrous substrates; however, by day 14, expression was significantly higher for cells cultured on RGDELP-coated nanofibrous substrates compared to uncoated nanofibrous substrates. Similarly, early $A L P$ mRNA expression was higher in uncoated nanofibrous substrates, but by day 21 , 
Fig. 3 Cells stained for ALP activity to determine osteogenic differentiation on uncoated monolayer substrates (a), uncoated nanofibrous substrates (b), RGD-ELP-coated monolayer substrates (c), RGD-ELP-coated nanofibrous substrates (d), FN-coated monolayer substrates (e), and FN-coated nanofibrous substrates (f). Scale bar $=500 \mu \mathrm{m}$



expression levels were significantly higher on RGD-ELPcoated nanofibrous substrates compared to both uncoated and FN-coated nanofibrous substrates. These data demonstrate that RGD-ELP coating enhances osteogenic differentiation.

The mRNA expressions of genes specific to chondrogenic differentiation, COL2, $A G N$, and $S O X 9$, were analyzed for MSCs cultured on nanofibrous substrates (Fig. 6d-f). Initial COL2 expression was highest in cells cultured on the uncoated nanofibrous substrates. By day 21, cells cultured on RGDELP-coated nanofibrous substrates exhibited COL2 expression levels significantly higher than those of cells cultured on FN-coated nanofibrous substrates and similar to those of cells cultured on uncoated nanofibrous substrates. $A G N$ expression was higher for cells cultured on uncoated and FNcoated nanofibrous substrates than for cells on RGD-ELPcoated nanofibrous substrates at days 7 and 14. Interestingly, SOX9 expression levels for cells cultured on RGD-ELPcoated nanofibrous substrates were higher than cells cultured on the other nanofibrous substrate conditions at day 7 . However, at days 14 and 21, SOX9 expression levels were similar between cells with all three nanofibrous substrate conditions. Taken together, these data indicate that chondrogenesis is not affected by RGD-ELP coating.
The mRNA expression level for PPARG and $L P L$ was analyzed to determine adipogenic induction (Fig. 6g, h). For both marker, the mRNA expression was significantly upregulated for cells cultured on uncoated and FN-coated nanofibrous substrates compared to cells cultured on RGD-ELP-coated nanofibrous substrates on day 7 . By day 14 , uncoated nanofibrous substrates maintained higher expression levels of both adipogenic genes compared to cells cultured with other nanofibrous substrate conditions, while cells cultured on FNcoated nanofibrous substrates had similar expression levels compared to RGD-ELP-coated nanofibrous substrates. However, by day 21, expression of both adipogenic genes was significantly higher for cells cultured on RGD-ELP-coated nanofibrous substrates compared to cells cultured on either of the other two nanofibrous substrates. These data indicate that the induction of adipogenesis was initially not effective on RGDELP-coated nanofibrous substrates but enhanced by day 21 .

\section{Discussion}

Investigation into the functional potential of ECM proteins to enhance stem cell adhesion, proliferation, and differentiation is 
Fig. 4 Osteogenic cells stained for Alizarin Red to visualize calcium nodule formation on uncoated monolayer substrates (a), uncoated nanofibrous substrates (b), RGD-ELP-coated monolayer substrates $(\mathbf{c})$, RGD-ELP-coated nanofibrous substrates (d), FN-coated monolayer substrates (e), and FN-coated nanofibrous substrates (f). Scale bar $=500 \mu \mathrm{m}$
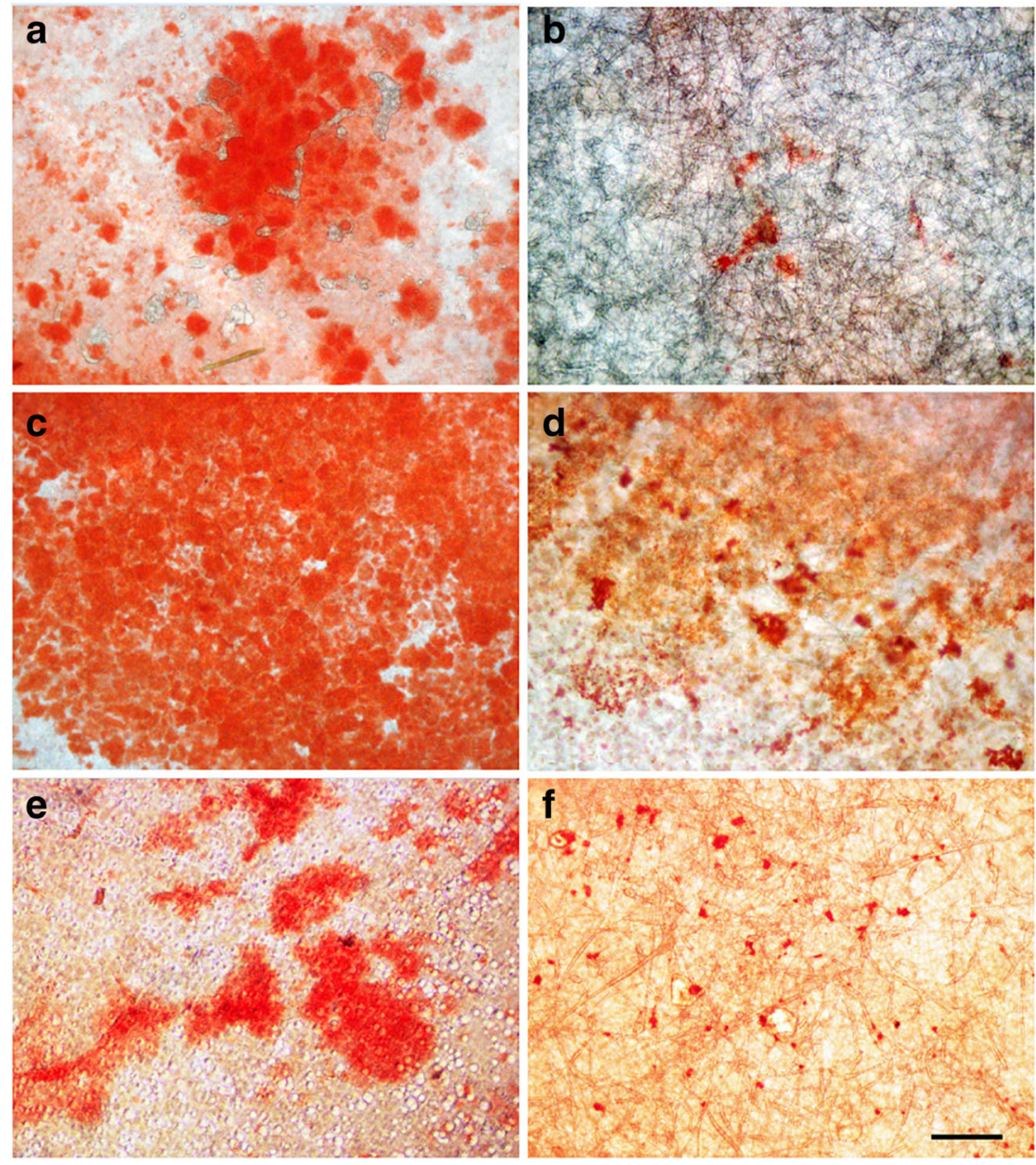

crucial for improving the design of biomaterials. A recombinant ECM peptide, ELP, which mimics the natural elastin peptide sequence, was recently developed. Most of the research on these peptides has previously focused on their use in drug delivery applications [26, 27]. However, several groups have shown promising findings in the use of ELP as an ECM component due to the ease with which its biological characteristics can be tuned $[28,29,31,35,40]$. One approach used to alter biological characteristics of recombinant ELP is the addition of other functional groups into the peptide sequence. Several other groups have manufactured RGD incorporating ELP similar to the peptide used in this study $[31,35]$. These previously examined RGD containing ELPs as well as some other ELP variations are of interest for stem cell applications. Thus far, MSCs have been cultured on ELP hydrogels, ELP-coated films, or ELP-coated tissue culture plastic and their adhesion, proliferation, and differentiation have been assessed [28, 29, 40, 41]. However, the effect of ELP coating on regulation of the cell cultured on a substrate with a structure that closely resembles the fibrous ECM such as collagen has not been examined. Our previous studies have shown that electrospun nanofibers structurally similar to collagen fibrils are able to promote multi-lineage differentiation of MSCs [15]. Particularly for osteogenesis, it has been demonstrated that the structural feature of nanofibers is capable of enhancing differentiation induction of MSCs [42]. Here, we examine adhesion, proliferation, and differentiation of MSCs on monolayer and nanofibrous substrates coated with RGD-ELP and report altered MSC morphology, enhanced MSC adhesion, and enhanced osteogenesis on RGD-ELP-coated nanofibrous substrates.

One interesting finding is that cell adhesion is enhanced on nanofibrous substrates coated with RGD-ELP compared to uncoated and $\mathrm{FN}$-coated substrates, but cell proliferation rates are lowest on RGD-ELP-coated nanofibrous substrates. Higher cell numbers were found on RGD-ELP-coated nanofibrous substrates initially, but by day 7 , cell numbers were similar between RGD-ELP-coated and FN-coated substrates and higher on uncoated substrates, indicating that RGD-ELP is not supportive of cell proliferation. Our data trends are similar to those reported in a study using poly $(\mathrm{N}$ isopropylacrylamide) (pNIPAM)-micropatterned surfaces with adsorbed ELP, where cells also adhered at a greater rate to the ELP-coated surface but did not display enhanced proliferation over time compared to pNIPAM substrates lacking ELP [41]. While Ozturk et al. have demonstrated similar patterns for adhesive and proliferative behavior of MSCs on ELP 
Fig. 5 Adipogenic cells stained for Oil Red O to visualize lipid droplet formation on uncoated monolayer substrates (a), uncoated nanofibrous substrates (b), RGD-ELP-coated monolayer substrates (c), RGD-ELP-coated nanofibrous substrates (d), FN-coated monolayer substrates (e), and FN-coated nanofibrous substrates (f). Scale bar $=500 \mu \mathrm{m}$. Quantification of oil droplets extracted from the fibrous substrates. ${ }^{*} p<0.05 ; n=3$. $U C$ uncoated substrates, $R G D$-ELP RGD-ELP-coated substrates, $F N$ fibronectin-coated substrates


and uncoated surfaces, it is still unclear why our RGD-ELP coating would have higher adhesive capabilities than FNcoated substrates. It is known that the addition of natural ECM components that contain the RGD peptide enhances both cell adhesion and proliferation [32-34, 43]. Therefore, it is not expected to find that cell adhesion is enhanced on RGD-ELP-coated substrates more so than on the FN-coated substrates since FN is known to also contain the RGD peptide. The likely cause of the difference in adhesion may be attributed to differences in the concentration of RGD peptides between RGD-ELP- and FN-coated substrates. In this study, $5 \mu \mathrm{g} / \mathrm{mL}$ of RGD-ELP or FN was used to coat substrates. With a difference in the molecular weights of RGD-ELP $(60.4 \mathrm{kDa})$ and FN (220 kDa) and each RGD-ELP monomer containing 20 RGD groups, the molar concentration of RGD in RGD-ELPcoated culture is $1.656 \mathrm{mM}$, approximately 73-fold higher than $22.73 \mu \mathrm{M}$ of RGD in FN-coated culture. Since increased surface density of this binding motif has been shown to enhance cell adhesion [43], the higher concentration of RGD peptide sequences on the RGD-ELP-coated substrate compared to the FN-coated substrate may result in enhanced cell adhesion on the RGD-ELP-coated substrate. However, this may not be 

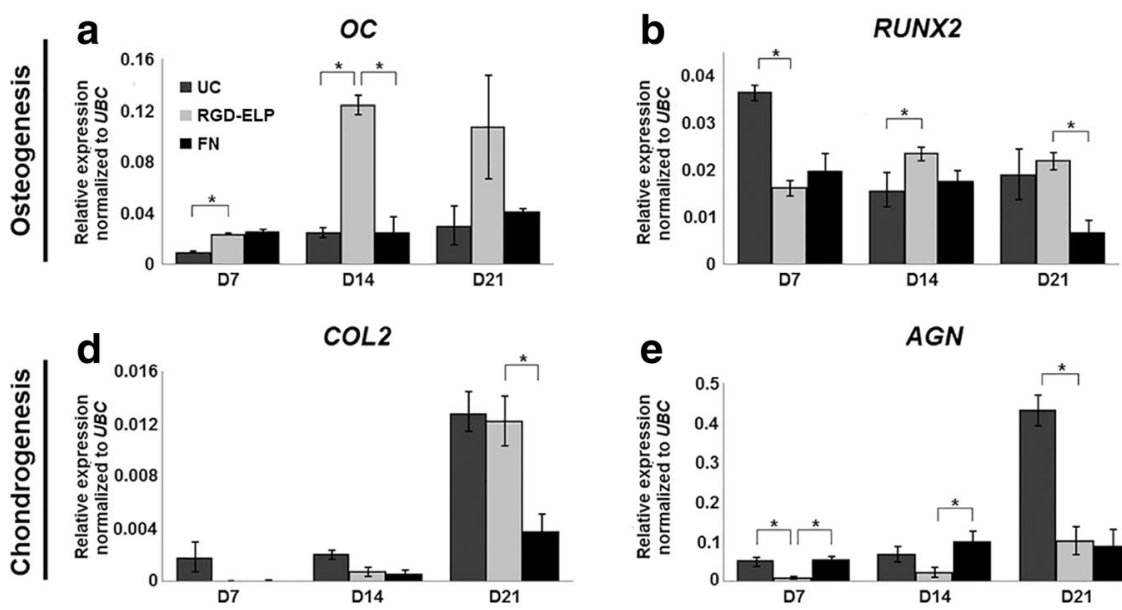

e

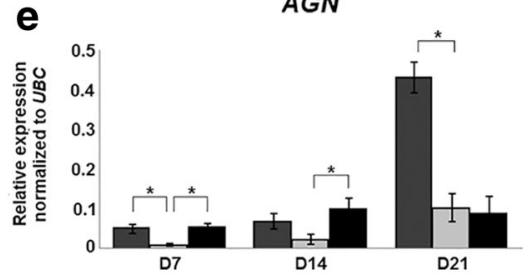

PPARG



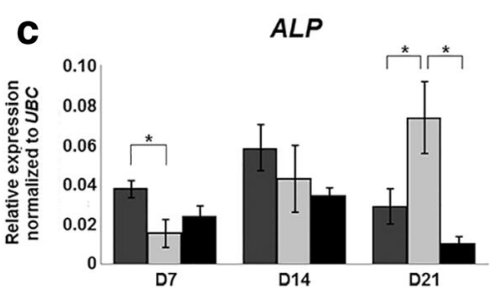

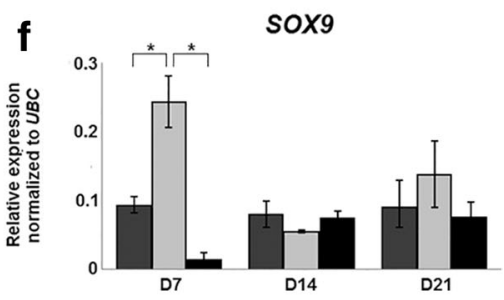

Fig. 6 Levels of mRNA expressions of bone-, cartilage-, and adipose-associated markers in MSCs cultured on nanofibrous substrates with or without RGD-ELP or FN coating. * $p<0.05 ; n=3$. UC uncoated substrates, $R G D$-ELP RGD-ELP-coated substrates, $F N$ fibronectin-coated substrates

able to fully explain different cell adhesion between RGD-ELPand FN-coated substrates because our previous study has also shown that cells have a higher affinity to the RGD motif of FN than to that of RGD-ELP [44]. Further studies are needed to investigate how the factors of concentration and affinity of RGD affect cell adhesion between RGD-ELP- and FN-coated substrates.

Interestingly, despite the presence of RGD adhesion peptides within both RGD-ELP and FN sequences, cell proliferation was greatest on the uncoated nanofibrous substrates. It is possible that the serum in our culture medium provided relevant binding proteins which adsorbed to the substrate surfaces over time. Different compositions of medium serums have been shown to differentially impact cell activities including proliferation [45]. Therefore, it is reasonable to consider that our specific lot of FBS could play a role in supporting proliferation. The presence of RGD-ELP or FN on the substrates would have blocked some adsorption of serum protein, whereas adsorption would be unrestricted on the uncoated substrates. Serum contains many different proteins which could stimulate cell growth more effectively than the RGD peptide, leading to enhanced cell proliferation compared to the coated substrates. Another possible explanation is that MSCs on RGD-ELP- or FN-coated substrates received induction signals from the coating and underwent spontaneous cell differentiation, which in turn reduced cell proliferation. However, this hypothesis needs to be further tested before a solid conclusion can be drawn.

The enhanced cell adhesion and decreased cell proliferation found on RGD-ELP-coated nanofibrous substrates were not found for cells cultured on monolayer RGD-ELPcoated substrates. On monolayer substrates, cell adhesion and proliferation were similar for uncoated, RGD-ELP-coated, and FN-coated substrates. There are two possible explanations for these differences. First, surface topography plays a role in directing stem cell behavior $[14,46]$. Therefore, it is not unexpected to find differences in cell adhesion and proliferation between cells cultured on monolayer and nanofibrous substrates. Secondly, protein adsorption and adsorbed protein conformation are altered by surface topography as well as dependent on the chemistry and stability of different proteins [47]. Changes in conformation result in altered active sites, meaning that cells might no longer interact with the protein in the same way. Thus, differences in surface topography likely result in different protein conformation and altered cell interactions with proteins, leading to differences in adhesion and proliferation seen between various monolayer and nanofibrous substrates.

On RGD-ELP-coated monolayer and nanofibrous substrates, cells maintained a spindle-like morphology with a higher aspect ratio than cells cultured on uncoated substrates. Of particular interest, cells cultured on RGD-ELP-coated nanofibrous substrates had similar morphology compared to cells cultured on FN-coated nanofibrous substrates. This indicates that the addition of RGD-ELP made synthetic substrates more favorable for maintaining phenotypic morphology of cells by providing adequate adhesion sites on substrates. Our data are in agreement with previous findings, suggesting that MSCs cultured on glass coverslips coated with ELP 
maintain an elongated morphology [28]. It is also known that a recombinant ECM peptide, such as RGD, is capable of regulating cell morphology [43]. Since our peptide contains both ELP and RGD sequences, it is likely that both of the sequences synergistically regulated cells to maintain the spindled, fibroblast-like morphology.

MSCs cultured on RGD-ELP-coated substrates demonstrate enhanced osteogenic differentiation compared to MSCs cultured on FN-coated or uncoated substrates. Our data suggest that RGD-ELP coating plays a stimulatory role in MSC osteogenesis. Different ECM proteins are known to influence cell behavior differently due to their specific integrinbinding sites and the resulting signal transduction pathways $[48,49]$. In addition, there is likely another factor of cell morphology that regulates cell differentiation as shown in our results demonstrating increased cell elongation on RGDELP-coated substrates compared to uncoated substrates. Elongated morphology and increased cytoskeletal tension are known to enhance osteogenesis [50, 51]. It is likely that the addition of RGD-ELP provided more cell adhesion sites on substrates for cell attachment, which in turn resulted in increased cytoskeletal tension in a cell. Changes in cell morphology caused by the addition of a biologically relevant peptide, RGD-ELP, may, at least in part, be involved in stimulating osteogenesis.

While osteogenesis was enhanced on substrates coated with RGD-ELP, chondrogenic and adipogenic differentiation overall were not enhanced. Adipogenic staining showed no difference in lipid droplet accumulation between RGD-ELPcoated and uncoated substrates regardless of the topography, and analysis of adipogenic marker expression showed mRNA upregulation on RGD-ELP-coated substrates only at the last time point. Analysis of mRNA expression of chondrogenic markers showed an increase in MSCs cultured on uncoated substrates compared to the cell on RGD-ELP-coated substrates. Interestingly, the results of a previous study have shown that chondrogenesis of adipose-derived stem cells is indeed supported when cultured in an ELP hydrogel [40]. The difference in chondrogenic support between the previous and our current findings is likely resulted from the variable in substrate structure rather than that in the presence of RGDELP. Hydrogels support a rounded morphology, while nanofibers coated with RGD-ELP support cell elongation along the fiber length. Cell morphology is important for directing cell fate, and both chondrogenesis and adipogenesis require cells to adopt a rounded morphology [50,52]. Differences in cell shape caused by the substrate structures likely resulted in the differences in chondrogenic differentiation seen in the two studies. Therefore, substrate architecture appears to play a more influential role is chondrogenic and adipogenic differentiation than does the presence of RGD-ELP.

Cells cultured on RGD-ELP-coated substrates demonstrate an elongated morphology and enhanced adhesion.
Differentiation along the osteogenic lineage was supported, but adipogenic and chondrogenic differentiation was not supported by RGD-ELP coating. To extend the study of how an RGD-ELP coating impacts cell differentiation, it might be interesting to examine MSC differentiation into tenocytes on RGD-ELP-coated substrates since elastin is a naturally occurring component of the tendon ECM [53]. While further studies are needed to understand the full potential of RGD-ELP as a surface coating, our study has already demonstrated effects of RGD-ELP on cell adhesion, proliferation, and osteogenic differentiation. Better understanding of how RGD-ELP impacts cell behavior will help us implement the peptide and other ECM molecules into substrates to better control cell differentiation.

\section{Conclusion}

The chemical and structural components of the ECM play a key role in directing stem cell fate. ELP is a recombinant peptide with repeating amino acid units which mimic the native ECM protein, elastin. Examination of the influence of RGD-ELP on MSC adhesion, proliferation, and differentiation reveals that RGD-ELP enhances cell adhesion, supports cell proliferation, and alters the cell morphology so that it adopts a more elongated morphology. When differentiation is induced, osteogenesis is enhanced on RGD-ELP-coated substrates. Cell differentiation is likely influenced by both the chemistry of RGD-ELP and the morphology cells adopted when cultured on RGD-ELP-coated substrates. Our findings have demonstrated the capabilities and limitations of RGDELP as a substrate surface coating and provided good understanding of how cells respond to this recombinant peptide.

Acknowledgments This study was supported by the Daegu Gyeongbuk Institute of Science and Technology grant 14-NB-01 and the Korea Ministry of Science, Information and Communication Technology and Future Planning Grant 2014R1A2A2A01005619.

\section{References}

1. Dominici M, Le Blanc K, Mueller I, Slaper-Cortenbach I, Marini F, Krause D, et al. Minimal criteria for defining multipotent mesenchymal stromal cells. The International Society for Cellular Therapy position statement. Cytotherapy. 2006;8:315-7.

2. Kolf C, Cho E, Tuan R. Biology of adult mesenchymal stem cells: regulation of niche, self-renewal, and differentiation. Arthritis Res Ther. 2007;9.

3. Pittenger M, Mackay A, Beck S, Jaiswal R, Douglas R, Mosca J, et al. Multilineage potential of adult human mesenchymal stem cells. Science. 1999;284:143-7.

4. Bonab M, Alimoghaddam K, Talebian F, Ghaffari S, Ghavamzdeh A, Nikbin B. Aging of mesenchymal stem cell in vitro. BMC Cell Biol. 2006;7. 
5. Stenderup K, Justesen J, Clausen C, Kassem M. Aging is associated with decreased maximal life span and accelerated senescence of bone marrow stromal cells. Bone. 2003;33:919-26.

6. Wagner W, Horn P, Castoldi M, Diehlmann A, Bork S, Saffrich R, et al. Replicative senescence of mesenchymal stem cells: a continuous and organized process. PLoS One. 2008;3.

7. Cheng H, Qiu L, Ma J, Zhang H, Cheng M, Li W, et al. Replicative senescence of human bone marrow and umbilical cord derived mesenchymal stem cells and their differentiation to adipocytes and osteoblasts. Mol Biol Rep. 2010;38:5161-8.

8. Kozhevnikova M, Mikaelyan A, Payushina O, Starostin V. Comparative characterization of mesenchymal bone marrow stromal cells at early and late stages of culturing. Biol Bull. 2008;35: $132-8$

9. Kern S, Eichler H, Stoeve J, Kluter H, Bieback K. Comparative analysis of mesenchymal stem cells from bone marrow, umbilical cord blood, or adipose tissue. Stem Cells. 2006;24:1294-301.

10. Lee R, Kim B, Choi I, Kim H, Choi H, Suh K, et al. Characterization and expression analysis of mesenchymal stem cells from human bone marrow and adipose tissue. Cell Physiol Biochem. 2004;14:311-24.

11. Musina R, Bekchanova E, Belyavskii A, Sukhikh G. Differentiation potential of mesenchymal stem cells of different origin. Bull Exp Biol Med. 2006;141:147-51.

12. Dalby M, Gadegaard N, Tare R, Andar A, Riehle M, Herzyk P, et al. The control of human mesenchymal cell differentiation using nanoscale symmetry and disorder. Nat Mater. 2007;6:997-1003.

13. Jiang X, Cao H, Shi L, Ng S, Stanton L, Chew S. Nanofiber topography and sustained biochemical signaling enhance human mesenchymal stem cell neural commitment. Acta Biomater. 2012;8: 1290-302.

14. McMurray R, Gadegaard N, Tsimbouri M, Burgess K, McNamara L, Tare R, et al. Nanoscale surfaces for the long-term maintenance of mesenchymal stem cell phenotype and multipotency. Nat Mater. 2011;10:637-44.

15. Li WJ, Tuli R, Huang X, Laqueriere P, Tuan RS. Multilineage differentiation of human mesenchymal stem cells in a threedimensional nanofibrous scaffold. Biomaterials. 2005;26:5158-66.

16. Prasad A, Berger D, Popat K. PCL nanopillars versus nanofibers: a contrast in progenitor cell morphology, proliferation, and fate determination. Adv Eng Mater. 2012;14:B351-B6.

17. Venugopal J, Low S, Choon A, Ramakrishna S. Interaction of cells and nanofiber scaffolds in tissue engineering. J Biomed Mater Res B Appl Biomater. 2007;84B:34-48.

18. Zhang Y, Quyang H, Lim C, Ramakrishna S, Huang Z. Electrospinning of gelatin fibers and gelatin/PCL composite fibrous scaffolds. J Biomed Mater Res B Appl Biomater. 2005;72B:156-65.

19. Amass W, Amass A, Tighe B. A review of biodegradable polymers: uses, current developments in synthesis and characterization of biodegradable polyesters, blends of biodegradable polymers and recent advances in biodegradation studies. Polym Int. 1999;47:89-144.

20. Badami A, Kreke M, Thompson M, Riffle J, Goldstein A. Effect of fiber diameter on spreading, proliferation, and differentiation of osteoblastic cells on electrospun poly(lactic acid) substrates. Biomaterials. 2006;27:596-606.

21. Cutright $\mathrm{D}$. The repair of fractures of the orbital floor using biodegradable polylactic acid. Oral Surg Oral Med Oral Pathol. 1972;33: 28-34.

22. Middleton J, Tipton A. Synthetic biodegradable polymers as orthopedic devices. Biomaterials. 2000;21:2335-46.

23. Place E, George J, Williams C, Stevens M. Synthetic polymer scaffolds for tissue engineering. Chem Soc Rev. 2009;38:1139-51.

24. Duan Y, Wang Z, Yan W, Wang S, Zhang S, Jia J. Preparation of collagen-coated electrospun nanofibers by remote plasma treatment and their biological properties. J Biomater Sci Polym Ed. 2007;18: 1153-64.

25. Seeger J, Klingman N. Improved endothelial cell seeding with cultured cells and fibronectin-coated grafts. J Surg Res. 1985;38:641-7.

26. Herrero-Vanrell R, Rincon A, Alonso M, Reboto V, MolinaMartinez I, Rodriguez-Cabello J. Self-assembled particles of an elastin-like polymer as vehicles for controlled drug release. J Control Release. 2005;102:113-22.

27. Meyer D, Shin B, Kong G, Dewhirst M, Chilkoti A. Drug targeting using thermally responsive polymers and local hyperthermia. J Control Release. 2001;74:213-24.

28. Ciofani G, Genchi GG, Liakos I, Athanassiou A, Mattoli V, Bandiera A. Human recombinant elastin-like protein coatings for muscle cell proliferation and differentiation. Acta Biomater. 2013;9:5111-21.

29. Celebi B, Cloutier M, Rabelo RB, Mantovani D, Bandiera A. Human elastin-based recombinant biopolymers improve mesenchymal stem cell differentiation. Macromol Biosci. 2012;12: 1546-54.

30. Tejeda-Montes E, Smith KH, Poch M, Lopez-Bosque MJ, Martin $\mathrm{L}$, Alonso $\mathrm{M}$, et al. Engineering membrane scaffolds with both physical and biomolecular signaling. Acta Biomater. 2012;8:998 1009.

31. Girotti A, Reguera J, Rodriguez-Cabello J, Arias F, Alonso M, Testera A. Design and bioproduction of a recombinant multi(bio)functional elastin-like protein polymer containing cell adhesion sequences for tissue engineering purposes. J Mater Sci Mater Med. 2004;15:479-84.

32. Kantlehner M, Schafner P, Finsinger D, Meyer J, Jonczyk A, Diefenbach B, et al. Surface coating with cyclic RGD peptides stimulates osteoblast adhesion and proliferation as well as bone formation. Chembiochem. 2000;1:107-14.

33. Lebaron R, Athanasiou K. Extracellular matrix cell adhesion peptides: functional applications in orthopedic materials. Tissue Eng. 2000;6:85-103.

34. Pierschbacher M, Ruoslahti E. Cell attachment activity of fibronectin can be duplicated by small synthetic fragments of the molecule. Nature. 1984;309:30-3.

35. Ravi S, Caves J, Martinez A, Haller C, Chaikof E. Incorporation of fibronectin to enhance cytocompatibility in multilayer elastin-like protein scaffolds for tissue engineering. J Biomed Mater Res A. 2013;101A:1915-25.

36. Tejeda-Montes E, Smith KH, Rebollo E, Gomez R, Alonso M, Rodriguez-Cabello JC, et al. Bioactive membranes for bone regeneration applications: effect of physical and biomolecular signals on mesenchymal stem cell behavior. Acta Biomater. 2014;10:134 41 .

37. Li W, Laurencin C, Caterson E, Tuan R, Ko F. Electrospun nanofibrous structure: a novel scaffold for tissue engineering. $\mathrm{J}$ Biomed Mater Res A. 2002;60:613-21.

38. Jeon W, Park B, Wei J, Park R. Stimulation of fibroblasts and neuroblasts on a biomimetic extracellular matrix consisting of tandem repeats of the elastic VGVPG domain and RGD motif. J Biomed Mater Res A. 2010;97A:152-7.

39. Pek Y, Wan A, Ying J. The effect of matrix stiffness on mesenchymal stem cell differentiation in a 3D thixotropic gel. Biomaterials. 2010;31:385-91.

40. Betre H, Ong S, Guilak F, Chikoti A, Fermon B, Setton L. Chondrocytic differentiation of human adipose-derived adult stem cells in elastin-like polypeptide. Biomaterials. 2006;27:91-9.

41. Ozturk N, Girotti A, Kose G, Rodriguez-Cabello J, Hasirci V. Dynamic cell culturing and its application to micropatterned, elastin-like protein-modified poly(N-isopropylacrylamide) scaffolds. Biomaterials. 2009;30:5417-26.

42. Chen W, Shao Y, Li X, Zhao G, Fu J. Nanotopographical surfaces for stem cell fate control: engineering mechanobiology from the bottom. Nano Today. 2014;9:759-84. 
43. Schuler M, Owen G, Hamilton D, de Wild M, Textor M, Brunette $\mathrm{D}$, et al. Biomimetic modification of titanium dental implant model surfaces using RGDSP-peptide sequence: a cell morphology study. Biomaterials. 2006;27:4003-15.

44. Jeon WB, Park BH, Choi SK, Lee KM, Park JK. Functional enhancement of neuronal cell behaviors and differentiation by elastinmimetic recombinant protein presenting Arg-Gly-Asp peptides. BMC Biotechnol. 2012;12:61.

45. Shahdadfar A, Fronsdal K, Haug T, Reinholt F, Brinchmann J. In vitro expansion of human mesenchymal stem cells: choice of serum is a determinant of cell proliferation, differentiation, gene expression, and transcriptome stability. Stem Cells. 2005;23: 1347-66.

46. Curtis A, Wilkinson C. Topographical control of cells. Biomaterials. 1997;18:1573-83.

47. Roach P, Farrar D, Perry C. Surface tailoring for controlled protein adsorption: effect of topography at the nanometer scale and chemistry. J Am Chem Soc. 2006;128:3939-45.
48. Clark E, Brugge J. Integrins ad signal transduction pathways: the road taken. Science. 1995;268:233-9.

49. Salasznyk R, Williams W, Boskey A, Batorsky A, Plopper G. Adhesion to vitronectin and collagen 1 promotes osteogenic differentiation of human mesenchymal stem cells. J Biomed Biotechnol. 2004;2004:24-34.

50. McBeath R, Pirone D, Nelson C, Bhadriraju K, Chen C. Cell shape, cytoskeletal tension, and RhoA regulate stem cell lineage commitment. Dev Cell. 2004;6:483-95.

51. Wang Y, Xiang Y, Cohen D, Wozniak M, Yang M, Lin G, et al. Bone morphogenetic protein-2-induced signaling and osteogenesis is regulated by cell shape, RhoA/ROCK, and cytoskeletal tension. Stem Cells Dev. 2012;21:1176-86.

52. Glowacki J, Trepman E, Folkman J. Cell shape and phenotypic expression in chondrocytes. Exp Biol Med. 1983;172:93-8.

53. James R, Kesturu G, Balian G, Chhabra B. Tendon: biology, biomechanics, repair, growth factors, and evolving treatment options. J Hand Surg. 2008;33:102-12. 